\title{
The reporting of progression criteria in protocols of pilot trials designed to assess the feasibility of main trials is insufficient: a meta-epidemiological study
}

\author{
Lawrence Mbuagbaw ${ }^{1,2,3^{*}}$ (D), Sarah Daisy Kosa ${ }^{1,4}$, Daeria O. Lawson ${ }^{1}$, Rosa Stalteri ${ }^{1}$, Oluwatobi R. Olaiya ${ }^{5}$,
} Ahlam Alotaibi ${ }^{6}$ and Lehana Thabane 1,2,7,8,9 $^{-1}$

\begin{abstract}
Introduction: Pilot and feasibility trials are conducted to determine feasibility or to collect information that would inform the design of a larger definitive trial. Clear progression criteria are required to determine if a definitive or main trial is feasible and how it should be designed. We sought to determine how often progression criteria are reported and the associated factors.

Methods: We conducted a methodological review of protocols for pilot randomised trials published in three journals that publish research protocols (BMJ Open, Trials, Pilot and Feasibility Studies), using a PubMed search (20132017). We extracted bibliometric information including the country in which the study was conducted, source of funding, type of intervention, use of a primary feasibility outcome, sample size reporting, and justification. We used generalised linear models to determine the factors associated with reporting progression criteria.

Results: Our search retrieved 276 articles, of which 49 were not eligible. We included 227 articles. Overall, 45/227 (19.8\%; 95\% confidence interval [CI] 14.8-25.6) reported progression criteria. Protocols published in more recent years were significantly associated with higher odds of reporting progression criteria (adjusted odds ratio [aOR] 1.40; 95\% Cl 1.03-1.92; $p=0.034$ ). Pilot trials from Europe (aOR 0.19; 95\% Cl 0.08-0.48; $p<0.001$ ) and the rest of the world (aOR $0.05 ; 95 \% \mathrm{Cl} 0.01-0.18 ; p<0.003$ ) compared to North America were significantly associated with lower odds of reporting progression criteria. Journal, source of funding, sample size, intervention type, and having a primary outcome related to feasibility were not significantly associated with reporting progression criteria.

Conclusion: Progression criteria are not often explicitly stated in protocols of pilot trials leaving room for varied interpretation of findings. The development of formal guidance for progression criteria in protocols of pilot trials is warranted.
\end{abstract}

Keywords: Pilot, Feasibility, Trials, Progression criteria, Protocols

\footnotetext{
* Correspondence: mbuagblc@mcmaster.ca

${ }^{1}$ Department of Health Research Methods, Evidence and Impact, McMaster

University, Hamilton, ON, Canada

${ }^{2}$ Biostatistics Unit, Father Sean O'Sullivan Research Centre, St Joseph's

Healthcare-Hamilton, 50 Charlton Avenue East, 3rd Floor Martha Wing,

Room H321, Hamilton, ON L8N 4A6, Canada

Full list of author information is available at the end of the article
}

(c) The Author(s). 2019 Open Access This article is distributed under the terms of the Creative Commons Attribution 4.0 International License (http://creativecommons.org/licenses/by/4.0/), which permits unrestricted use, distribution, and reproduction in any medium, provided you give appropriate credit to the original author(s) and the source, provide a link to the Creative Commons license, and indicate if changes were made. The Creative Commons Public Domain Dedication waiver (http://creativecommons.org/publicdomain/zero/1.0/) applies to the data made available in this article, unless otherwise stated. 


\section{Background}

Pilot and feasibility studies are increasingly being used to inform the feasibility and design of larger trials [1]. They may be used to test procedures, instruments, and techniques that would be applied in a main study [2]. They help to provide useful information on the processes required to implement the trial, resources required, management issues, and scientific information (safety, dosing, treatment effect, etc.) [3]. Though often used interchangeably, [1] feasibility and pilot studies are not synonymous. Feasibility studies encompass the broad range of studies that address concerns about feasibility, and include randomised pilot studies, non-randomised pilot studies, and other types of non-pilot feasibility studies [4]. In this paper, we focused on small-scale randomised trials designed to inform the conduct of a future larger trial. We make no distinction on whether they are internal (integral and structurally similar to the main trial) or external (meant to provide information that will determine the structure of the main trial).

The value of pilot studies is increasingly being recognised. The UK Medical Research Council, the Canadian Institutes for Health Research (CIHR), and the US National Institutes for Health $(\mathrm{NIH})$ all recommend the use of pilot studies to inform larger trials [5-7].

They are not meant to provide definitive information on treatment effects, and therefore, hypothesis testing is discouraged [1, 8]. Likewise, sample size estimates drawn from pilot studies may be misleading, given that they are often very small [3]. Many studies are pointing out concerns with how pilot studies are conducted and reported. For example, Arain et al. found that many pilot studies inappropriately focus on hypothesis testing [1]. Duffet et al. found that pilot trials in the paediatric literature focus on clinical outcomes and rarely justify their sample sizes or report criteria for success [9]. The abstracts of pilot trials in heart failure were found to be poorly reported [10]. Other authors have noted that in very few reports of pilot studies is it stated that they were conducted in preparation for a larger trial [11].

Current guidance suggests that sample size estimations for pilot studies may be done in a variety of ways depending on whether it is an internal or an external pilot study, some based on rules of thumb, the nature of the outcomes (continuous or binary), others based on the confidence interval approach or as a fraction of the fully powered large trial $[3,12,13]$. Pilot studies are generally small, but small studies should not be labelled as pilot studies if they are not pilot studies.

Given these concerns, recent efforts such as the Consolidated Standards of Reporting Trials (CONSORT) extension for pilot trials have outlined recommended approaches for reporting pilot trials [14]. As precursors to larger trials, pilot studies are expected to provide information on whether a larger trial is feasible and if so how it should be designed. Other authors have suggested strategies to select, interpret, and apply progression criteria (criteria that inform the decision to progress to a larger definitive trial) [15]. While it is expected that these criteria be reported in the pilot study manuscript, it is also important that these progression criteria be pre-specified at the protocol stage. In fact, it is recommended that these progression criteria be agreed upon by the funders and investigators [15]. Statistical approaches to informing progression have also been suggested [16]. Often, there is no detailed outline of the decision-making process that would lead to stopping, amending, or proceeding to a larger trial, [15] as such it is unclear whether the decision to continue with a larger trial was determined a priori or post hoc.

Avery and colleagues propose a traffic light system for specifying progression criteria for internal pilot studies, where green (go) indicates that the criteria have been met and the trial should proceed, amber (amend) indicates that some changes should be made to the larger trial, and red (stop) indicates that the investigators should not move forward with the larger trial [15]. Some examples of the application of progression criteria include the following: a pilot trial of strategies to enhance venous thromboprophylaxis in which the investigators deemed the trial to be definitely feasible if $\geq 70 \%$ of eligible patients completed the risk assessment form [17], and a pilot trial of rituximab for non-splenectomized patients with immune thrombocytopaenia in which the progression criteria include the recruitment of at least 60 patients in 12 months and successful blinding of staff, among others [18].

The research protocol is the ideal opportunity for investigators to report key methodological issues including the use and interpretation of progression criteria. However, there is currently no guidance on how to report a protocol for a pilot trial. In order to inform the use and interpretation of progression criteria among trialists and other stakeholders, we therefore sought to investigate the use of progression criteria in protocols of pilot trials.

We hypothesised that the use of progression criteria might be associated with certain study characteristics. For example, reporting quality improves over time, journals have different editorial policies that influence the nature of the final publication, research capacity varies by country, source of funding and study size might be indicative of the resources (including methods scientists) available to complete the study, and the type of intervention (pharmacological versus not pharmacological) may be linked to funding and may play a role in how information is reported. These study characteristics have been found to be associated with reporting in other studies [19]. 


\section{Objectives}

Our objectives were to describe reporting of progression criteria to main trial and to determine the factors associated with reporting of progression criteria.

\section{Methods}

\section{Design}

We conducted a methodological review of protocols of pilot studies published in the past 5 years (2013-2017) in three journals known to publish research protocols: British Medical Journal (BMJ) Open, Pilot and Feasibility Studies (PAFS), and Trials. All three journals are indexed in PubMed. We applied the following search strategy, including terms for the journals of interest, protocol, pilot or feasibility, and time limits (01 January 2013 to 31 December 2017):

\section{((BMJ Open [Journal] OR Pilot Feasibility Stud [Journal] OR Trials [Journal])) AND (Pilot [Title] OR Feasibility [Title] AND Protocol [Title])}

\section{Data management}

The full text of all identified citations was screened for eligibility. The eligibility criteria were as follows: (1) published in one of the three journals of interest, (2) a protocol for a pilot randomised trial, and (3) within the time range 2013-2017. Data were extracted by one reviewer and verified by a second independent reviewer. Agreement statistics were not captured. We extracted the following data: bibliographic information (author, year, and journal); country of origin, study objectives, main outcome measures or feasibility criteria, and presence of progression criteria; source of funding; and sample size estimation and justification for sample size.

When the planned sample size was reported as a range of values, the median was taken. When different sample sizes were reported for the different participants (e.g. health workers, patients, carers), we used the sample size for those who would be randomised. We categorised the studies as small or large, based on the median sample size of all the studies. The country in which the pilot was planned was collected and reorganised into world regions to facilitate analyses. We grouped the justifications for sample size as inadequate (based on intervention effect size, other similar studies, or no justification given) or adequate (based on a feasibility outcome, a proportion of the larger trial, and recommendations in literature).

Study data were collected and managed using the Research Electronic Data Capture (REDCap) tool hosted at St Joseph's Healthcare Hamilton. REDCap is a secure, web-based application designed to support data capture for research studies, providing an intuitive interface for validated data entry; audit trails for tracking data manipulation and export procedures; automated export procedures for seamless data downloads to common statistical packages; and procedures for importing data from external sources [20].

\section{Analysis}

First, data were summarised descriptively as counts (percentages) in cross tabulations according to whether they reported progression criteria.

Second, we used generalised linear models to determine the relationship between reporting of progression criteria (yes/no) and study characteristics. We assumed a binomial distribution (applying the logit link). The covariates were entered as a block: journal (PAFS, BMJ Open, and Trials), year of publication (continuous), source of funding (industry or government/private), sample size (small [0-60], large [>60]), region (North America, Europe, other), primary outcome related to feasibility (yes/no), and intervention type (pharmacological versus non-pharmacological). These variables have been shown to be associated with reporting standards [19]. The level of significance was set at $\alpha=0.05$. Model fit was assessed using Akaike's information criterion (AIC), comparing a full model with all the predictors and a reduced model with selected predictors. Crude odds ratio (OR) and adjusted odds ratios (aOR), corresponding 95\% confidence intervals $(\mathrm{CI})$, and $p$ values are reported. Data were analysed using the $\mathrm{glm}$ command in Stata 15 (StataCorp, 2017. Stata Statistical Software: Release 15. College Station, TX: StataCorp LLC) [21].

\section{Results}

Our search retrieved 276 studies, of which 49 were not eligible (21 were protocols for non-randomised studies, 19 were full reports, 7 were errata or corrigenda, 1 was a methodological paper, and 1 was a trial update). Of the 227 included studies, only 45 (19.8\%, 95\% CI 14.8-25.6) reported progression criteria. Our screening process is outlined in Fig. 1.

Almost half $(50.2 \%)$ of the studies came from Trials and were conducted in Europe (52.9\%). More studies were published in 2017 than in any other year (31.7\%). The other characteristics of the included studies are reported in Table 1.

The proportion of studies reporting progression criteria by key study characteristics are outlined in Table 2.

Table 3 includes the results of both unadjusted univariate and adjusted multivariable analyses (model 1 and model 2). After multivariable adjustment, more recent year of publication (adjusted odds ratio [aOR] 1.40; 95\% CI 1.03-1.92; $p=0.034$ ) was associated with reporting progression criteria. Pilot trials from Europe (aOR 0.19; 95\% CI $0.08-0.48 ; p<0.001)$ and the rest of the world 


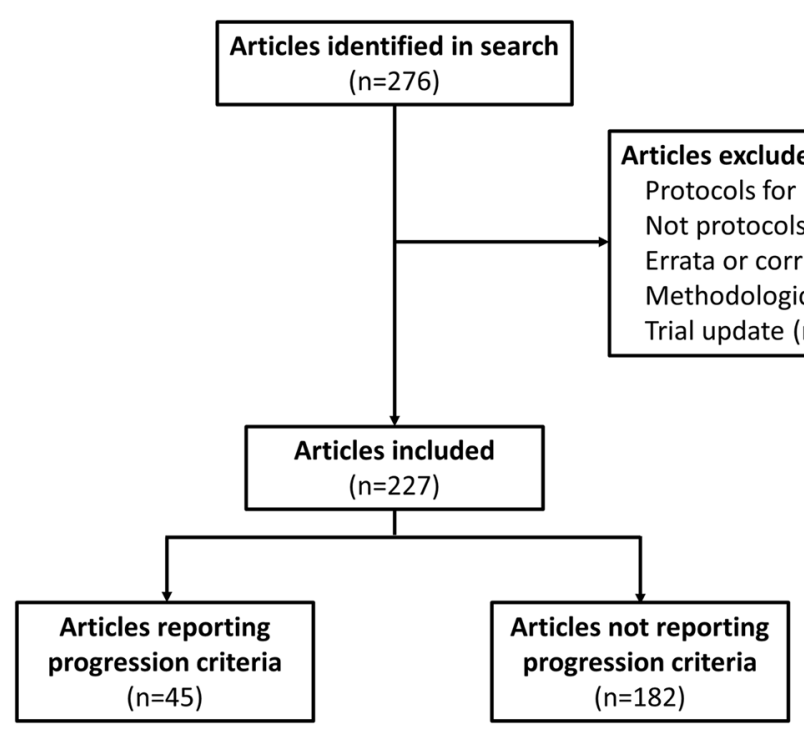

Fig. 1 Flow chart of study selection

(aOR $0.05 ; 95 \%$ CI $0.01-0.18 ; p<0.003$ ) compared to North America were significantly associated with lower odds of reporting progression criteria. Journal, source of funding, sample size, and having a primary outcome related to feasibility were not associated with reporting progression criteria in this model (model 1). In a reduced model (model 2), excluding source of funding, sample size, intervention type, and feasibility outcomes, articles from Europe (aOR 0.22; 95\% CI 0.10-0.49; $p<$ $0.001)$ and the rest of the world (aOR 0.04; $95 \% \mathrm{CI}$ $0.01-0.15 ; p<0.001)$ were less likely to report progression criteria. See Table 3.

\section{Discussion}

In this methodological review, we have shown that at the planning stages of pilot trials (i.e. in published protocols), progression criteria are not often reported, and sample sizes not often adequately justified. The use of progression criteria appears to be associated with some study characteristics of the manuscript such as journal and region of publication.

To the best of our knowledge, this is the first methodological review of protocols for pilot trials and highlights some important concerns in the design and reporting of these protocols. The small number (19.8\%; 95\% CI 14.8-25.6) reporting clear progression criteria is concerning, given that progression criteria are required to determine how the results of feasibility will be interpreted. If progression criteria are not set a priori, there is a risk that some studies that did not do well in the pilot stage may be moved to a larger trial without modification or due acknowledgement of potential limitations. On the other hand, successful pilot trials or trials with amenable concerns may not proceed to larger trials if interpretation of success is subjective.

We identified a few study level characteristics that were associated with the use of progression criteria. Each of these characteristics has previously been shown to be associated with reporting quality of trials in general.

Journal characteristics including endorsement of specific reporting standards and impact factor influence the nature of published reports. This has been shown in other methodologic reviews in which journals endorsing the CONSORT statement or requiring its use and higher impact factor journals published papers with better reporting [22-24]. In this study, we did not find the journal of publication to be associated with progression criteria.

As researchers develop more tailored guidance for reporting research and journals endorse these reporting standards, it can be expected that reporting will improve over time. Other studies have shown that reporting improves over time [25, 26]. More recent studies were more likely to report progression criteria only in our univariate analyses and multivariable analyses.

Larger studies tend to have better reporting quality $[25,27,28]$. Study size is probably a reflection of the resources available to complete the study. These resources would include methodological support and therefore better reporting of key methodological issues. Other studies have previously highlighted some differences when statisticians are involved, such as better interpretation of negative trials, [29] sample size calculation, and computations for multiple endpoints [30]. We did not find any association between study size and reporting of progression criteria. 
Table 1 Characteristics of included studies

\begin{tabular}{|c|c|}
\hline Variable & Number (\%) \\
\hline Overall & $227(100)$ \\
\hline \multicolumn{2}{|l|}{ Journal } \\
\hline PAFS* & $42(18.5)$ \\
\hline BMJ Open** & $71(31.1)$ \\
\hline Trials & $114(50.2)$ \\
\hline \multicolumn{2}{|l|}{ Year of publication } \\
\hline 2013 & $34(15.0)$ \\
\hline 2014 & $37(16.3)$ \\
\hline 2015 & $34(15.0)$ \\
\hline 2016 & $50(22.0)$ \\
\hline 2017 & $72(31.7)$ \\
\hline \multicolumn{2}{|l|}{ Region } \\
\hline North America & $43(18.9)$ \\
\hline Europe & $120(52.9)$ \\
\hline Other & $64(28.2)$ \\
\hline \multicolumn{2}{|l|}{ Funding } \\
\hline Industry & $52(24.5)$ \\
\hline Government or private & $160(75.5)$ \\
\hline \multicolumn{2}{|l|}{ Intervention type } \\
\hline Pharmacological & $35(15.4)$ \\
\hline Non-pharmacological & $191(84.1)$ \\
\hline Feasibility outcomes (yes) & $123(54.2)$ \\
\hline Sample size reported (yes) & $220(96.9)$ \\
\hline \multicolumn{2}{|l|}{ Sample size } \\
\hline Small $(n=0-60)$ & $143(64.7)$ \\
\hline Large $(n>60)$ & 78 (35.3) \\
\hline \multicolumn{2}{|l|}{ Sample size justification } \\
\hline Adequate & $99(44.8)$ \\
\hline Inadequate & $122(55.2)$ \\
\hline
\end{tabular}

*Pilot and Feasibility Studies

**British Medical Journal

The role of region in reporting of science is unclear and may have to do with differential research methods capacity and the use of English as a native language. For example, other methodological papers have shown better reporting in non-Chinese reports, compared to those from China, [31] and in North American and UK reports, compared to Scandinavia and other countries [25]. In this study, North American and European studies were more likely to have progression criteria reported.

Source of funding also influences reporting, sometimes in favour of industry-funded trials or non-industryfunded trials [27, 32, 33]. In this study, we did not find any association between funding and reporting of progression criteria.
Table 2 Distribution of studies that reported progression criteria

\begin{tabular}{|c|c|}
\hline Variable & Number (\%); $95 \% \mathrm{Cl}{ }^{*}$ \\
\hline Overall & 45 (19.8); $14.8-25.6$ \\
\hline \multicolumn{2}{|l|}{ Journal } \\
\hline PAFS** & $9(20.0) ; 9.6-34.6$ \\
\hline BMJ Open ${ }^{* * *}$ & $16(35.6) ; 21.9-51.2$ \\
\hline Trials & $20(44.4) ; 29.6-60.0$ \\
\hline \multicolumn{2}{|l|}{ Year of publication } \\
\hline 2013 & $4(8.9) ; 2.5-21.2$ \\
\hline 2014 & $3(6.7) ; 1.3-18.3$ \\
\hline 2015 & $5(11.1) ; 3.7-24.1$ \\
\hline 2016 & $19(42.2) ; 27.6-57.8$ \\
\hline 2017 & $14(31.1) ; 18.2-46.7$ \\
\hline \multicolumn{2}{|l|}{ Region } \\
\hline North America & $21(46.7) ; 31.6-62.13$ \\
\hline Europe & $21(46.7) ; 31.6-62.13$ \\
\hline Other & $3(6.7) ; 1.3-18.3$ \\
\hline \multicolumn{2}{|l|}{ Funding } \\
\hline Industry & $12(28.6) ; 14.6-41.9$ \\
\hline Government or private & $30(71.4) ; 51.1-80.0$ \\
\hline \multicolumn{2}{|l|}{ Intervention type } \\
\hline Pharmacological & $7(15.6) ; 6.5-29.5$ \\
\hline Non-pharmacological & $37(82.2) ; 67.9-92.0$ \\
\hline Feasibility outcomes (yes) & $29(64.4) ; 48.8-78.13$ \\
\hline Sample size reported (yes) & 45 (100); 92.13-100.00 \\
\hline \multicolumn{2}{|l|}{ Sample size } \\
\hline Small $(n=0-60)$ & $29(64.4) ; 48.8-78.1$ \\
\hline Large $(n>60)$ & $16(35.6) ; 21.9-51.2$ \\
\hline \multicolumn{2}{|l|}{ Sample size justification } \\
\hline Adequate & $28(62.2) ; 46.5-76.2$ \\
\hline Inadequate & 17 (37.8); 23.7-53.5 \\
\hline
\end{tabular}

${ }^{*}$ Confidence interval for percentage

**Pilot and Feasibility Studies

***Bitish Medical Journal

Even though other studies have noted better reporting of pharmacological intervention studies, [34] we did not find any association.

Sample size justification in pilot studies is a subject of debate. While it is generally agreed that a calculation is not always required, there must be a reason for including a particular number of people in a study for ethical, scientific, and economic reasons. The literature includes numerous approaches to estimating sample size which we considered to all be some form of justification [12, 13, 35]. A complete absence of justification or using the sample size from a previous study were both considered inadequate.

Previous research has shown that building pilot studies around clinical or efficacy outcomes instead of feasibility outcomes (as the main outcome) is associated with 
Table 3 Factors associated with using progression criteria

\begin{tabular}{|c|c|c|c|c|c|c|}
\hline \multirow[t]{2}{*}{ Variable } & \multicolumn{2}{|l|}{ Univariate model } & \multicolumn{2}{|c|}{ Multivariable model 1} & \multicolumn{2}{|c|}{ Multivariable model 2} \\
\hline & OR $(95 \% \mathrm{Cl})$ & $p$ & $\mathrm{aOR}(95 \% \mathrm{Cl})$ & $p$ & aOR $(95 \% \mathrm{Cl})$ & $p$ \\
\hline \multicolumn{7}{|l|}{ Journal } \\
\hline PAFS & 1 & & 1 & & 1 & \\
\hline BMJ Open & $1.07(0.42-2.69)$ & 0.891 & $2.43(0.68-8.61)$ & 0.170 & $1.97(0.69-5.58)$ & 0.204 \\
\hline Trials & $0.78(0.32-1.88)$ & 0.581 & $1.67(0.49-5.56)$ & 0.414 & $1.33(0.48-3.68)$ & 0.588 \\
\hline Year of publication & $1.30(1.02-1.65)$ & 0.037 & $1.40(1.03-1.92)$ & 0.034 & $1.34(1.00-1.80)$ & 0.050 \\
\hline \multicolumn{7}{|l|}{ Source of funding } \\
\hline Industry & 1 & & 1 & & Not in model & \\
\hline Government or private & $0.77(0.36-1.64)$ & 0.497 & $0.72(0.26-1.99)$ & 0.532 & Not in model & \\
\hline \multicolumn{7}{|l|}{ Sample size } \\
\hline Small $(n=0-60)$ & 1 & & 1 & & Not in model & \\
\hline Large $(n>60)$ & $1.01(0.51-2.01)$ & 0.967 & $1.27(0.58-2.79)$ & 0.549 & Not in model & \\
\hline \multicolumn{7}{|l|}{ Region } \\
\hline North America & 1 & & 1 & & 1 & \\
\hline Europe & $0.22(0.08-0.62)$ & 0.004 & $0.19(0.08-0.48)$ & $<0.001$ & $0.22(0.10-0.49)$ & $<0.001$ \\
\hline Other & $0.05(0.03-0.09)$ & $<0.001$ & $0.05(0.01-0.18)$ & $<0.001$ & $0.04(0.01-0.15)$ & $<0.001$ \\
\hline \multicolumn{7}{|l|}{ Intervention type } \\
\hline Non-pharmacological & 1 & & 1 & & Not in model & \\
\hline Pharmacological & $1.01(0.41-2.49)$ & 0.977 & $0.79(0.27-2.36)$ & 0.396 & Not in model & \\
\hline \multicolumn{7}{|l|}{ Feasibility outcomes } \\
\hline Yes & 1 & & 1 & & Not in model & \\
\hline No & $0.59(0.31-1.11)$ & 0.100 & $0.95(0.43-2.10)$ & 0.862 & Not in model & \\
\hline AIC & Not applicable & & 0.933 & & 0.880 & \\
\hline
\end{tabular}

PAFS Pilot and Feasibility Studies, BMJ British Medical Journal, OR unadjusted odds ratio, aOR adjusted odds ratio, AIC Akaike's information criterion

worse reporting [36]. In this study, we did not find any association between having a primary feasibility outcome and reporting progression criteria. This may be because some studies reported progression criteria based on secondary feasibility criteria, even though the primary outcome was based on a clinical/efficacy outcome.

We advise some caution in the interpretation of our findings: first, because we acknowledge the relative novelty of discussions around the use of progression criteria, their importance, and how they should be reported, and second, the absence of formal guidance or reporting standards for their use. In addition, there may be other journals that publish protocols of pilot studies that we were unaware of. However, this work adds to the growing list of methodological concerns with pilot trials [1, 9-11, 36] and highlights areas for improvement. Further, there may be interaction between some of the study characteristics. For example, some journals have been publishing for longer than others, and researchers in certain regions may have preferences for certain journals. In cross tabulations, we found significant associations between journal and year (no studies from PAFS in 2013 and 2014; more studies from Trials across all years) and between journal and region (BMJ Open mostly publishing from the rest of the world and Trials mostly publishing papers from Europe). These issues could be investigated further in other studies but are unlikely to be unique to pilot studies.

As such, we recommend the development of formal guidance on the design and reporting of protocols of pilot trials, and that protocols of pilot studies clearly indicate what information will inform the decision to move to a larger trial, without which the pilot does not fulfil its purpose.

\section{Conclusion}

Progression criteria are not often reported in protocols of pilot trials. There is room for the development of formal guidance and recommendations on the use of progression criteria in pilot randomised trials. Investigators should outline a list of feasibility criteria, how they will be interpreted, and how this interpretation will inform progression to a larger trial. The consequences of not using progression criteria, including ill-informed large trials, are enough justification to warrant a closer look at pilot studies with no explicit progression criteria. 


\section{Abbreviations}

aOR: Adjusted odds ratio; BMJ: British Medical Journal; Cl: Confidence intervals; CIHR: Canadian Institutes for Health Research; CONSORT: Consolidated Standards of Reporting Trials; GEE: Generalised estimation equations; NIH: National Institutes for Health; OR: Odds ratio; PAFS: Pilot and feasibility studies; REDCap: Research Electronic Data Capture; UK: United Kingdom

\section{Acknowledgements}

None

\section{Authors' contributions}

LM and LT designed the study. LM wrote the first draft. LM and RS analysed the data. RS, SDK, OO, AA, and DL extracted the data and revised several versions of the manuscript. All authors reviewed the final version of the manuscript. All authors read and approved the final manuscript.

\section{Funding}

No funding was used for this study.

\section{Availability of data and materials}

The datasets used and/or analysed during the current study are available from the corresponding author on reasonable request.

\section{Ethics approval and consent to participate}

Not applicable.

\section{Consent for publication}

Not applicable.

\section{Competing interests}

The authors declare that they have no competing interests.

\section{Author details}

${ }^{1}$ Department of Health Research Methods, Evidence and Impact, McMaster University, Hamilton, ON, Canada. ${ }^{2}$ Biostatistics Unit, Father Sean O'Sullivan Research Centre, St Joseph's Healthcare-Hamilton, 50 Charlton Avenue East, 3rd Floor Martha Wing, Room H321, Hamilton, ON L8N 4A6, Canada. ${ }^{3}$ Centre for the Development of Best Practices in Health, Yaoundé, Cameroon. ${ }^{4}$ Toronto General Hospital, University Health Network, Toronto, ON, Canada. ${ }^{5}$ Michael G. DeGroote School of Medicine, McMaster University, Hamilton, ON, Canada. ${ }^{6}$ Department of Pediatrics, McMaster University, Hamilton, ON, Canada. ${ }^{7}$ Departments of Paediatrics and Anaesthesia, McMaster University, Hamilton, ON, Canada. ${ }^{8}$ Centre for Evaluation of Medicine, St Joseph's Healthcare-Hamilton, Hamilton, ON, Canada. ${ }^{9}$ Population Health Research Institute, Hamilton Health Sciences, Hamilton, ON, Canada.

\section{Received: 19 December 2018 Accepted: 16 September 2019}

\section{Published online: 03 November 2019}

\section{References}

1. Arain M, Campbell MJ, Cooper CL, Lancaster GA. What is a pilot or feasibility study? A review of current practice and editorial policy. BMC Med Res Methodol. 2010;10:67.

2. Hassan ZA, Schattner P, Mazza D. Doing a pilot study: why is it essential? Malays Fam Physician. 2006;1:70-3.

3. Thabane L, Ma J, Chu R, Cheng J, Ismaila A, Rios LP, Robson R, Thabane M, Giangregorio L, Goldsmith $\mathrm{CH}$. A tutorial on pilot studies: the what, why and how. BMC Med Res Methodol. 2010:10:1.

4. Eldridge SM, Lancaster GA, Campbell MJ, Thabane L, Hopewell S, Coleman $\mathrm{CL}$, Bond $\mathrm{CM}$. Defining feasibility and pilot studies in preparation for randomised controlled trials: development of a conceptual framework. PLoS One. 2016:11:e0150205

5. Craig P, Dieppe P, Macintyre S, Michie S, Nazareth I, Petticrew M. Developing and evaluating complex interventions: the new Medical Research Council guidance. Bmj. 2008;337:a1655.

6. Tri-Council Policy Statement: Ethical Conduct for Research Involving Humans. http://www.pre.ethics.gc.ca/eng/policy-politique_tcps2-eptc2_201 8.html. Accessed 29 Oct 2019.

7. Pilot Studies: Common Uses and Misuses. https://nccih.nih.gov/grants/ whatnccihfunds/pilot_studies\#targetText=The $\% 20$ most $\% 20$ common $\% 2$
Omisuses\%20of,of\%20the\%20larger\%20scale\%20study. Accessed 29 Oct 2019.

8. Leon AC, Davis LL, Kraemer HC. The role and interpretation of pilot studies in clinical research. J Psychiatr Res. 2011;45:626-9.

9. Duffett M, Choong K, Hartling L, Menon K, Thabane L, Cook DJ. Pilot randomized trials in pediatric critical care: a systematic review. Pediatr Crit Care Med. 2015;16:e239-44.

10. Isiguzo GC, Zunza M, Chirehwa M, Mayosi BM, Thabane L. Quality of pilot trial abstracts in heart failure is suboptimal: a systematic survey. Pilot Feasibility Stud. 2018:4:107.

11. Lancaster GA, Dodd S, Williamson PR. Design and analysis of pilot studies: recommendations for good practice. J Eval Clin Pract. 2004;10:307-12.

12. Sandvik L, Erikssen J, Mowinckel P, Rodland EA. A method for determining the size of internal pilot studies. Stat Med. 1996;15:1587-90.

13. Teare MD, Dimairo M, Shephard N, Hayman A, Whitehead A, Walters SJ. Sample size requirements to estimate key design parameters from external pilot randomised controlled trials: a simulation study. Trials. 2014;15:264.

14. Eldridge SM, Chan CL, Campbell MJ, Bond CM, Hopewell S, Thabane L, Lancaster GA. CONSORT 2010 statement: extension to randomised pilot and feasibility trials. Pilot Feasibility Stud. 2016;2:64.

15. Avery KNL, Williamson PR, Gamble C, Connell Francischetto E, Metcalfe C, Davidson P, Williams H, Blazeby JM. Informing efficient randomised controlled trials: exploration of challenges in developing progression criteria for internal pilot studies. BMJ Open. 2017;7(2):e013537.

16. Hampson LV, Williamson PR, Wilby MJ, Jaki T. A framework for prospectively defining progression rules for internal pilot studies monitoring recruitment. Stat Methods Med Res. 2018:27(12):3612-27.

17. Pai M, Lloyd NS, Cheng J, Thabane L, Spencer FA, Cook DJ, Haynes RB, Schünemann HJ, Douketis JD. Strategies to enhance venous thromboprophylaxis in hospitalized medical patients (SENTRY): a pilot cluster randomized trial. Implement Sci. 2013:8:1.

18. Arnold DM, Heddle NM, Carruthers J, Cook DJ, Crowther MA, Meyer RM, Liu Y, Cook RJ, McLeod A, MacEachern JA, et al. A pilot randomized trial of adjuvant rituximab or placebo for nonsplenectomized patients with immune thrombocytopenia. Blood. 2012;119:1356-62.

19. Samaan Z, Mbuagbaw L, Kosa D, Borg Debono V, Dillenburg R, Zhang $S$, Fruci $V$, Dennis B, Bawor $M$, Thabane $L$. A systematic scoping review of adherence to reporting guidelines in health care literature. J Multidiscip Healthc. 2013;6:169-88.

20. Harris PA, Taylor R, Thielke R, Payne J, Gonzalez N, Conde JG. Research electronic data capture (REDCap)--a metadata-driven methodology and workflow process for providing translational research informatics support. J Biomed Inform. 2009;42:377-81.

21. StataCorp L. Stata multilevel mixed-effects reference manual. College Station: StataCorp LP; 2013.

22. Areia M, Soares M, Dinis-Ribeiro M. Quality reporting of endoscopic diagnostic studies in gastrointestinal journals: where do we stand on the use of the STARD and CONSORT statements? Endoscopy. 2010;42: 138-47.

23. Capili B, Anastasi JK, Geiger JN. Adverse event reporting in acupuncture clinical trials focusing on pain. Clin J Pain. 2010;26:43-8.

24. Montane E, Vallano A, Vidal X, Aguilera C, Laporte JR. Reporting randomised clinical trials of analgesics after traumatic or orthopaedic surgery is inadequate: a systematic review. BMC Clin Pharmacol. 2010;10:2.

25. Farrokhyar F, Chu R, Whitlock R, Thabane L. A systematic review of the quality of publications reporting coronary artery bypass grafting trials. Can J Surg. 2007:50:266-77.

26. Mbuagbaw L, Thabane M, Vanniyasingam T, Borg Debono V, Kosa S, Zhang S, Ye C, Parpia S, Dennis BB, Thabane L. Improvement in the quality of abstracts in major clinical journals since CONSORT extension for abstracts: a systematic review. Contemp Clin Trials. 2014;38:245-50.

27. Rios LP, Odueyungbo A, Moitri MO, Rahman MO, Thabane L. Quality of reporting of randomized controlled trials in general endocrinology literature. J Clin Endocrinol Metab. 2008;93:3810-6.

28. Thabane L, Chu R, Cuddy K, Douketis J. What is the quality of reporting in weight loss intervention studies? A systematic review of randomized controlled trials. Int J Obes. 2007:31:1554-9.

29. Peron J, You B, Gan HK, Maillet D, Chen EX, Pond GR. Influence of statistician involvement on reporting of randomized clinical trials in medical oncology. Anti-Cancer Drugs. 2013;24:306-9. 
30. Jaykaran, Chavda N, Yadav P. Proper reporting of statistical parameters in clinical trials published in indian medical journals. Is inclusion of statistician play any significant role? J Young Pharm. 2011;3:167-8.

31. Zhong Y, Zhou W, Jiang H, Fan T, Diao X, Yang H, Min J, Wang G, Fu J, Mao B. Quality of reporting of two-group parallel randomized controlled clinical trials of multi-herb formulae: A survey of reports indexed in the Science Citation Index Expanded. European Journal of Integrative Medicine. 2011; 3(4):e309-16.

32. Chowers MY, Gottesman BS, Leibovici L, Pielmeier U, Andreassen S, Paul M. Reporting of adverse events in randomized controlled trials of highly active antiretroviral therapy: systematic review. J Antimicrob Chemother. 2009;64: 239-50.

33. de Vries TW, van Roon EN. Low quality of reporting adverse drug reactions in paediatric randomised controlled trials. Arch Dis Child. 2010;95:1023-6.

34. Sriganesh K, Bharadwaj S, Wang M, Abbade LPF, Jin Y, Philip M, Couban R, Mbuagbaw $L$, Thabane L. Quality of abstracts of randomized control trials in five top pain journals: a systematic survey. Contemp Clin Trials Commun. 2017;7:64-8.

35. Cocks K, Torgerson DJ. Sample size calculations for pilot randomized trials: a confidence interval approach. J Clin Epidemiol. 2013;66:197-201.

36. Shanthanna H, Kaushal A, Mbuagbaw L, Couban R, Busse J, Thabane L. A cross-sectional study of the reporting quality of pilot or feasibility trials in high-impact anesthesia journals. Can J Anesth. 2018;65(11):1180-95.

\section{Publisher's Note}

Springer Nature remains neutral with regard to jurisdictional claims in published maps and institutional affiliations.

Ready to submit your research? Choose BMC and benefit from:

- fast, convenient online submission

- thorough peer review by experienced researchers in your field

- rapid publication on acceptance

- support for research data, including large and complex data types

- gold Open Access which fosters wider collaboration and increased citations

- maximum visibility for your research: over $100 \mathrm{M}$ website views per year

At BMC, research is always in progress.

Learn more biomedcentral.com/submissions 